\title{
Heterotopia in Individuals with 22q11.2 Deletion Syndrome
}

\author{
(D)E. Neuhaus, (DE. Hattingen, (D) S. Breuer, (DE. Steid,, (D) N. Polomac, (DF. Rosenow, (D). Rüber, (DE. Herrmann, (DC. Ecker, \\ (D) Kushan, (D) A. Lin, (D) A. Vajdi, (D) C.E. Bearden, and (D) A. Jurcoane
}

\section{ABSTRACT}

BACKGROUND AND PURPOSE: MR imaging studies and neuropathologic findings in individuals with 22q11.2 deletion syndrome show anomalous early brain development. We aimed to retrospectively evaluate cerebral abnormalities, focusing on gray matter heterotopia, and to correlate these with subjects' neuropsychiatric impairments.

MATERIALS AND METHODS: Three raters assessed gray matter heterotopia and other morphologic brain abnormalities on 3D TIWI and T2* WI in 75 individuals with 22q11.2 deletion syndrome (27 females, 15.5 [SD, 7.4] years of age) and 53 controls (24 females, 12.6 [SD, 4.7] years of age). We examined the association among the groups' most frequent morphologic findings, general cognitive performance, and comorbid neuropsychiatric conditions.

RESULTS: Heterotopia in the white matter were the most frequent finding in individuals with 22q11.2 deletion syndrome $(n=29$; controls, $n=0$; between-group difference, $P<.001)$, followed by cavum septi pellucidi and/or vergae $(n=20$; controls, $n=0$; $P<.001)$, periventricular cysts $(n=10$; controls, $n=0 ; P=.007)$, periventricular nodular heterotopia $(n=10$; controls, $n=0$; $P=.007$ ), and polymicrogyria ( $n=3$; controls, $n=0 ; P=.3$ ). However, individuals with these morphologic brain abnormalities did not differ significantly from those without them in terms of general cognitive functioning and psychiatric comorbidities.

CONCLUSIONS: Taken together, our findings, periventricular nodular heterotopia or heterotopia in the white matter (possibly related to interrupted Arc cells migration), persistent cavum septi pellucidi and/or vergae, and formation of periventricular cysts, give clues to the brain development disorder induced by the 22 q11.2 deletion syndrome. There was no evidence that these morphologic findings were associated with differences in psychiatric or cognitive presentation of the 22q11.2 deletion syndrome.

ABBREVIATIONS: $\mathrm{ADHD}=$ attention deficit/hyperactivity disorder; $\mathrm{ASD}=$ autism spectrum disorder; $\mathrm{CSP}=$ cavum septi pellucidi; $\mathrm{CV}=$ cavum vergae; 22 q11.2DS $=22$ q11.2 deletion syndrome; $\mathrm{IQ}=$ intelligence quotient; $\mathrm{PNH}=$ periventricular nodular heterotopia

$\mathbf{T}$ 22q11.2 deletion syndrome (22q11.2DS, also known as velocardiofacial or DiGeorge syndrome) is the most common microdeletion syndrome, which occurs in $\sim 1$ in $3000-$ 6000 live births. ${ }^{1}$ Phenotypically, individuals with 22q11.2DS may show a variety of symptoms, including congenital heart defects, velopharyngeal insufficiency, and immunodeficiency. ${ }^{1}$

Received April 9, 2021; accepted after revision July 19.

From the Institute of Neuroradiology (E.N., E. Hattingen, S.B., E.S., N.P., A.J.), Department of Neurology and Epilepsy Center Frankfurt Rhine-Main (E.N., F.R., T.R.), LOEWE Center for Personalized Translational Epilepsy Research (E.N., F.R., T.R.), Institute of Biostatistics and Mathematical Modelling (E. Herrmann), and Department of Child and Adolescent Psychiatry, Psychosomatics and Psychotherapy (C.E.), Goethe University Frankfurt, Frankfurt am Main, Germany; Department of Epileptology (T.R.), University Hospital Bonn, Bonn, Germany; Department of Forensic and Neurodevelopmental Sciences, Institute of Psychiatry, Psychology and Neuroscience (C.E.), King's College, London, UK; Department of Psychiatry and Biobehavioral Sciences (L.K., A.L., A.V., C.E.B.), Semel Institute for Neuroscience and Human Behavior, and Department of Psychology (C.E.B.), University of California, Los Angeles, Los Angeles, California.

This work was supported by the National Institute of Mental Health R01 MH085953, IR21MH116473-01A1, and 1U01MH119736-01 (to C.E.B.).
22q11.2DS is also associated with an elevated risk of psychiatric disorders such as attention deficit/hyperactivity disorder (ADHD), anxiety disorders, autism spectrum disorder (ASD), schizophrenia during adulthood, ${ }^{2}$ and epilepsy, ${ }^{3,4}$ as well as intellectual disability. ${ }^{5}$ To date, structural neuroimaging studies have focused mainly on group differences between individuals with 22q11.2DS and controls in terms of brain volume $e^{6,7}$ and white matter structural changes. ${ }^{8}$ Subjects with 22q11.2DS show widespread volume reductions in multiple gray and white matter regions, particularly in the midline ${ }^{6,7}$ and major

Paper previously presented, in part, as an e-poster at: Annual Conference of the German Society for Neuroradiology-neuroRAD, October 8-9, 2020; Virtual.

Please address correspondence to Elisabeth Neuhaus, MD, Institute of Neuroradiology, University Hospital Frankfurt, Schleusenweg 2-16, 60528 Frankfurt am Main, Germany; e-mail: elisabeth.neuhaus@kgu.de

- Indicates open access to non-subscribers at www.ajnr.org

Indicates article with online supplemental data.

http://dx.doi.org/10.3174/ajnr.A7283 
Table 1: Summary of psychiatric and cognitive assessment by group

\begin{tabular}{lccc}
\hline \multicolumn{1}{c}{ Characteristic } & Controls $(\boldsymbol{n}=\mathbf{5 3})$ & 22q11.2DS $(\boldsymbol{n}=\mathbf{7 5})$ & $\boldsymbol{P}$ Value \\
\hline Mean verbal IQ T-score (SD) & $59(13)$ & $36(9)$ & $<.001^{\mathrm{a}}$ \\
Mean nonverbal IQ T-score (SD) & $54(11)$ & $35(11)$ & $<.001^{\mathrm{a}}$ \\
Mean full-scale IQ (SD) & $112(20)$ & $78(12)$ & $<.001^{\mathrm{a}}$ \\
ASD (No.) (\%) & $0(0 \%)$ & $14(19 \%)$ & $<.001^{\mathrm{b}}$ \\
ADHD (No.) (\%) & $3(5.7 \%)$ & $38(51 \%)$ & $<.001^{\mathrm{b}}$ \\
Psychotic disorder (No.) (\%) & $0(0 \%)$ & $4(5.3 \%)$ & $.14^{\mathrm{b}}$ \\
Anxiety disorders (No.) (\%) & $8(15 \%)$ & $37(49 \%)$ & $<.001^{\mathrm{b}}$ \\
\hline
\end{tabular}

aWilcoxon rank sum test.

${ }^{b}$ Fisher exact test.

corticocortical white matter connections. ${ }^{8}$ Neuropathologic reports ${ }^{9,10}$ found evidence that the deletion may disrupt neurodevelopment, leading to altered migration of neurons in early developmental stages, which, in turn, results in impaired connectivity or organization of the fiber tracts. Morphologic MR imaging analyses of the brain supported this hypothesis, reporting, among other findings, polymicrogyria ${ }^{11}$ and enlarged Sylvian fissures, ${ }^{12}$ both indicating a migration disorder during brain development.

Another common malformation of cortical development is periventricular nodular heterotopia $(\mathrm{PNH})$. It is formed by ectopic aggregates of neurons, caused by interruption of neuronal migration from the ventricles to their correct location in the cortex. ${ }^{13}$ To our knowledge, only 2 studies found evidence of $\mathrm{PNH}$ in 22q11.2DS. One had a small sample size, ${ }^{10}$ and the other had a clinical focus. ${ }^{4}$ This gap in knowledge prompted us to evaluate and report MR imaging morphologic abnormalities of the brain focused on detection of PNH in a large cohort of 75 individuals with molecularly confirmed 22q11.2DS and in 53 controls. In addition, we investigated the impact of the most frequent neuroradiologic abnormalities found in this study on general cognitive performance and neuropsychiatric conditions.

\section{MATERIALS AND METHODS Subjects}

This is a retrospective analysis of a data set from an originally prospectively acquired DTI and volumetric study at the University of California, Los Angeles. The local ethics committee approved the study, and written informed consent or assent for minors was obtained for all participants. The sample consisted of 80 subjects with molecularly confirmed 22q11.2DS and 57 controls.

Exclusion criteria were neurologic or medical conditions (unrelated to 22q11.2DS) that might affect the brain structure, a history of head injury, insufficient fluency in English, and/or substance or alcohol abuse or dependence within the past 6 months. Controls additionally were excluded if they had intellectual disability or met the criteria for any major mental disorder except ADHD or a past episode of depression. ${ }^{14}$

Nine subjects were excluded from analysis due to motion artifacts. Thus, analyzable data consisted of 75 subjects with 22q11.2DS (37 females, 38 males; mean age, 15.5 [SD, 7.4] years) and 53 controls (24 females, 29 males; mean age, 12.6 [SD, 4.7] years) with a $P$ value of .7 for sex and .05 for age.

\section{Psychiatric, Cognitive, and Neurologic Assessment}

At the time of MR imaging, all subjects underwent psychiatric and cognitive testing (Structured Clinical Interview for the Diagnostic and Statistical Manual of Mental Disorders and/or Computerized Diagnostic Interview for Children). General intellectual functioning was assessed using the Wechsler Abbreviated Scale of Intelligence or Wechsler Adult Intelligence Scale, 4th ed. The diagnosis of ASD was based on the Autism Diagnostic Observation Schedule ${ }^{15}$ and the Autism Diagnostic Interview-Revised. ${ }^{16}$ A complete description of the battery is published elsewhere. ${ }^{14}$ These assessments are detailed in Table 1. As expected based on prior literature, ${ }^{2,5}$ individuals with 22q11.2DS had significantly lower intelligence quotients (IQs) and higher rates of diagnoses of developmental neuropsychiatric disorders. Seizure history was determined by parent interviews. One individual with 22q11.2DS had an epilepsy history and was taking an anticonvulsant medication; 2 individuals had a history of absence seizures in early childhood but no confirmed epilepsy diagnosis. All others had no seizure history. Due to the mere anamnestic survey and the small number of individuals with epilepsy, we have not performed any analyses regarding epilepsy.

\section{Image Acquisition and Analyses}

MRIs were acquired on 2 identical 3T MR imaging systems (Trio; Siemens) using a 12-channel head coil. A whole-brain sagittal 3D MPRAGE sequence was acquired with the following parameters: $\mathrm{TR} / \mathrm{TE} / \mathrm{TI}=2300 / 2.9 / 900 \mathrm{~ms}$, flip angle $=9^{\circ}$, matrix size $=240 \times$ 256, in-plane resolution $=1 \times 1 \mathrm{~mm}, 160$ slices, thickness = $1.2 \mathrm{~mm}$. A T2* sequence was acquired with the following parameters: $\mathrm{TR} / \mathrm{TE}=5000 / 33 \mathrm{~ms}$, matrix size $=128 \times 128$, in-plane resolution $=1.64 \times 1.64 \mathrm{~mm}$, 35 slices, thickness $=3 \mathrm{~mm}$.

Three experienced board-certified neuroradiologists (S.B., E. Hattingen, and E.S.) independently evaluated the MR images, blinded to the presence of 22q11.2DS and without having any background information on 22q11.2DS. The first and second raters identified obvious gray matter heterotopia-like lesions (cortexisointense, round, or ovoid) on T1WI. During their analyses, they also noticed additional morphologic findings: cavum septi pellucidi (CSP) and/or vergae (CV), periventricular cysts, and polymicrogyria. Then, they confirmed heterotopia and cysts by consensus on $\mathrm{T} 2{ }^{\star} \mathrm{WI}$ : heterotopia as isointense to cortex and cysts as isointense to CSF. T2 ${ }^{\star}$ WI was not available for 2 subjects. The third rater independently analyzed the data with respect to the additional morphologic findings, and the final evaluation was performed by consensus by the 3 raters.

The association between the most common morphologic findings in this study and general cognitive performance or comorbid neuropsychiatric conditions was investigated.

\section{Statistical Methods}

All analyses were performed in the $\mathrm{R}$ statistical environment (Version 3.6; http://www.r-project.org/). ${ }^{17}$ Differences in demographics, psychiatric and cognitive assessment, and frequency of 
Table 2: Frequency of morphologic anomalies in the 2 groups

\begin{tabular}{lccc}
\hline \multicolumn{1}{c}{ Characteristic } & Controls $(\boldsymbol{n}=\mathbf{5 3})$ & 22q11.2DS $(\boldsymbol{n}=\mathbf{7 5})$ & $\boldsymbol{P}$ Value \\
\hline Any morphologic finding & $0(0 \%)$ & $48(64 \%)$ & $<.001$ \\
Heterotopia & & & \\
Any localization & $0(0 \%)$ & $34(45 \%)$ & $<.001$ \\
PNH & $0(0 \%)$ & $10(13 \%)$ & .007 \\
Heterotopia in the WM & $0(0 \%)$ & $29(39 \%)$ & $<.001$ \\
CSP and/or CV & $0(0 \%)$ & $20(27 \%)$ & $<.001$ \\
Isolated CSP & $0(0 \%)$ & $1(1.3 \%)$ & $>.9$ \\
CSP/CV & $0(0 \%)$ & $19(25 \%)$ & $<.001$ \\
Cysts & $0(0 \%)$ & $10(13 \%)$ & .007 \\
Polymicrogyria & $0(0 \%)$ & $3(4.0 \%)$ & .30 \\
\hline
\end{tabular}

Note:-FDR indicates false discovery rate.

${ }^{\text {a }}$ Fisher exact test.

morphologic anomalies between controls and individuals with 22q11.2DS were tested using the Wilcoxon rank sum test or Fisher exact test. To assess whether certain morphologic findings are more likely to occur together in individuals with 22q11.2DS, we performed the Fisher exact test pair-wise. The conditional maximum likelihood estimate was used to estimate the odds ratios. Cognitive test results of individuals with 22q11.2DS with and without morphologic findings were compared using the 2-sample $t$ test or Wilcoxon rank sum test. The Shapiro-Wilk test was used for normality testing; and the Fisher F-test, for the equality of variances. A multivariate logistic regression was performed to test the associations between morphologic findings and the prevalence of psychiatric disorders in individuals with 22q11.2DS.

\section{RESULTS}

\section{Morphologic Findings}

Of the 75 individuals with 22q11.2 DS, 48 (64\%) had morphologic findings versus none of the 53 controls $(0 \% ; P<.001)$ (Table 2$)$.

Specifically, in this sample, we found the following morphologic abnormalities: heterotopia (periventricular or in the white matter), periventricular cysts, polymicrogyria, and CSP and/or CV (Fig 1 and Table 2).

The heterotopia-like lesions identified on T1WI were also isointense to the cortex on the $\mathrm{T} 2^{\star} \mathrm{WI}$ in all individuals with $22 \mathrm{q} 11.2 \mathrm{DS}$ and were thus confirmed as heterotopia. The periventricular cystlike lesions identified on T1WI were isointense to the CSF in all except 1 T2*WI (Online Supplemental Data). In 1 subject, a cystlike lesion proved to be a $\mathrm{PNH}$ by additional review of the $\mathrm{T} 2{ }^{\star} \mathrm{WI}$. T2 ${ }^{\star}$ WI was not available for 1 individual with $\mathrm{PNH}$ and 1 with a cyst. Heterotopia and cysts occurred mostly in the frontal regions (10/10 PNH, 28/29 heterotopia in the white matter, 9/10 cysts). In 7/29 individuals with 22q11.2DS, the distribution of heterotopia in the white matter exceeded that in the frontal region. heterotopialike lesions in the white matter were also found in 4 controls on the T1WI. However, these proved to be isointense to the CSF on $\mathrm{T} 2{ }^{*} \mathrm{WI}$, so they are most likely enlarged perivascular spaces and not heterotopia (Online Supplemental Data).

Heterotopia and cysts frequently appeared together (Online Supplemental Data; not significant after false discovery rate adjustment). Other findings appeared independently. PNH were in all except 2 individuals, multiple $(n=2-13)$ and bilateral. The cysts were bilateral in 7 individuals, and 6 individuals had multiple cysts $(n=2-$ 12). A single cyst or heterotopion was adjacent to the anterior body of the lateral ventricle. When multiple, they appeared arranged in arching, chainlike structures in the sagittal view (Fig 1). In $19 / 20$ individuals with 22q11.2DS, the CSP occurred in combination with CV; in 1 individual, CSP occurred alone. Polymicrogyria was unilateral, rightsided, and located in the peri-Sylvian area in all 3 individuals (Fig 1).

\section{Association with Cognition, Psychiatric Disorders, and Neurology}

There were no significant associations between morphologic findings and general cognitive performance for heterotopia (Fig 2), other single findings, or all findings summed together (Online Supplemental Data).

Multivariate logistic regression (Online Supplemental Data) showed no significant association between heterotopia and psychiatric diagnoses (Fig 3) or between other morphologic findings and psychiatric diagnoses.

The subject with confirmed epilepsy had no morphologic abnormalities.

\section{DISCUSSION}

We retrospectively examined morphologic abnormalities in a large, originally prospectively assessed cohort of 75 subjects with 22q11.2DS and 53 typically developing controls. We found a significantly higher rate of anomalous morphologic findings in those with 22q11.2DS (64\%) than in controls (0\%).

We found a significantly elevated rate of $\mathrm{PNH}$ in subjects with 22 q11.2DS, with a prevalence of $13 \%$ versus $0 \%$ in controls. A few studies have so far estimated the prevalence of PNH in 22q11.2DS: A study in a sample of 195 subjects (referred for a clinical MR imaging scan) found a $4.1 \%$ prevalence, ${ }^{4}$ and a study with a sample of 29 subjects (recruited from an epilepsy genetics clinic) found a $17.2 \%$ prevalence. ${ }^{10}$ Given the originally prospective assessment of our cohort, our prevalence estimate is not biased by clinical referral. In our cohort, all PNH are uncommonly located on top of the dorsal pole of the frontal horn of the lateral ventricles, which is a pattern also reported by Rezazadeh et al. ${ }^{10}$ In contrast, "classic" PNH found in other conditions are bilateral, multiple, nearly contiguous, and aligned along the outline of the lateral ventricle walls. ${ }^{13}$ Additionally, we identified heterotopia in the white matter with a prevalence of $39 \%$. Heterotopic neurons or nodules in the frontal white matter were described in some neuropathologic case reports of individuals with 22q11.2DS but were, in these cases, only microscopically visible. ${ }^{9,10}$

A DTI study found impaired connectivity, which the authors attributed, among other causes, to ectopic neurons in the white matter. ${ }^{8}$ In a study investigating epileptic seizures in individuals with 22q11.2DS, 1 subject with unilateral frontal white matter heterotopion was mentioned, ${ }^{18}$ suggesting that the heterotopia visible on MR imaging may be only the tip of the iceberg. The literature describes an accumulation of nonspecific white matter lesions ${ }^{19,20}$ in individuals with 22q11.2DS with the same localization and shape as the heterotopia we found in the white matter. 

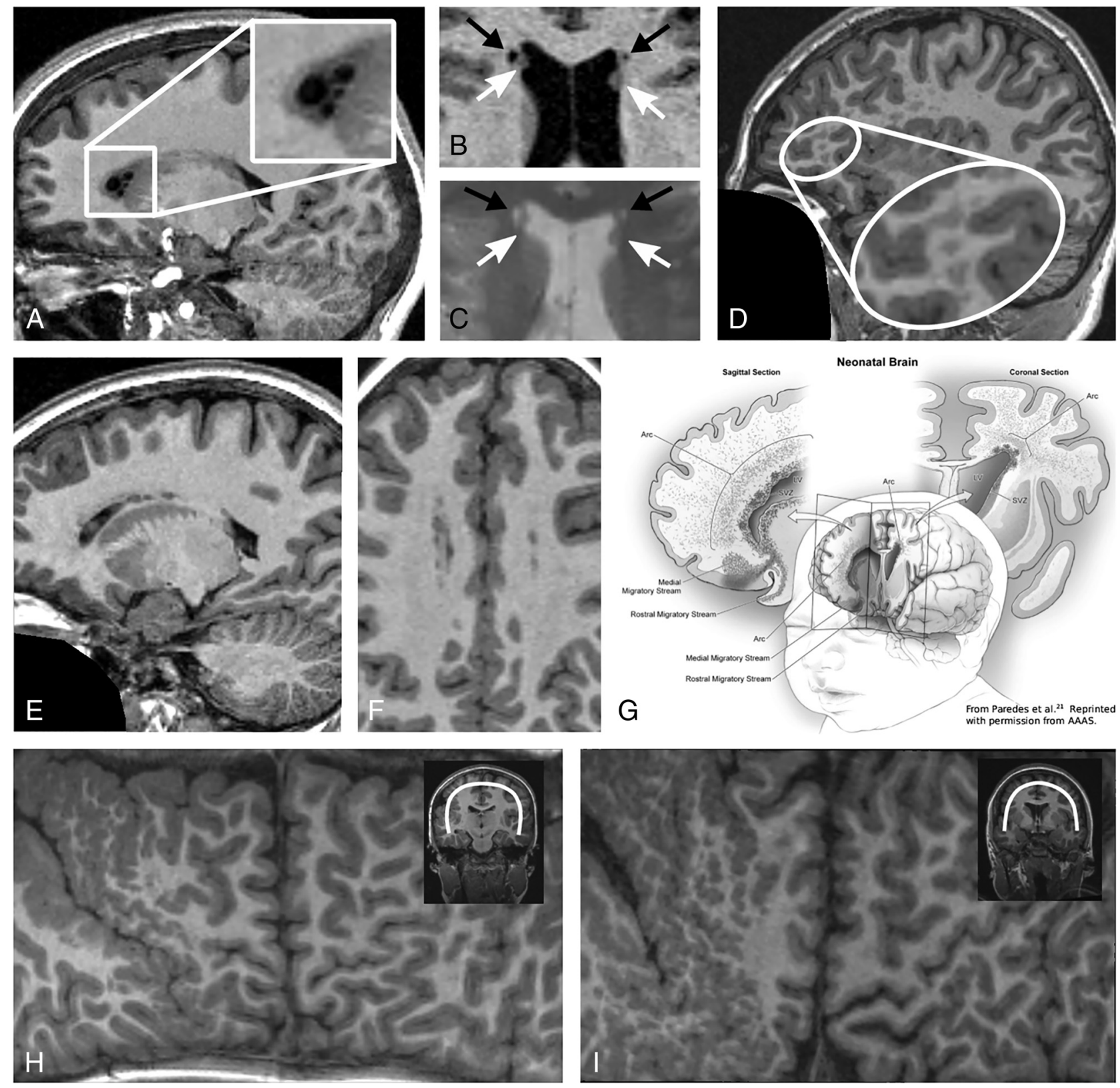

FIG 1. Representative examples of morphometric findings in brain MRls of 6 subjects with 22q11.2DS. Subject 1: Small cysts adjacent to the anterior horns of the lateral ventricles $(A)$. Subject 2: TIWI $(B)$ and T2*WI $(C)$ of bilateral cysts (black arrows, isointense to CSF in both sequences) and periventricular heterotopia (white arrows, cortex-isointense in both sequences). Subject 3: Multiple, perivascular, and frontal heterotopia in the white matter $(D)$. Subject 4: Multiple periventricular heterotopia forming an arcuate structure in the sagittal view with a remarkable overlap with the migratory stream of the Arc cells outlined in $G(E, F$, and $G)$. The drawing in $G$ is taken from Paredes et al ${ }^{21}$ (reprinted with permission from American Association for the Advancement of Science), who were the first to identify Arc cells as a population of late-moving neurons at this localization. Subjects 5 and 6: Exemplary Mercator brain projections of 2 of the 3 individuals with polymicrogyria in the peri-Sylvian area of the right hemisphere $(H$ and $I)$.

The occurrence in the frontal white matter supports the hypothesis of Rezazadeh et $\mathrm{al}^{10}$ that heterotopia in $22 \mathrm{q} 11.2 \mathrm{DS}$ might result from arrested Arc migrating cells. Arc cells are a population of late-migrating neurons, identified by Paredes et al. ${ }^{21}$ These neurons form a caplike structure surrounding the anterior body of the lateral ventricle or an arching structure in sagittal sections in the postnatal infant human brain (Fig 1). They continue to migrate along radial glial fibers tangentially to the walls of the lateral ventricles and along blood vessels into the anterior cingulate gyrus and prefrontal cortex in the early postnatal period, when they differentiate and contribute to inhibitory circuits. Paredes et al ${ }^{21}$ describe Arc cells as histopathologically organized into 4 tiers from the subventricular zone to the cortex. This distribution of Arc cells led Rezazadeh et al to the hypothesis that the PNH they observed were Arc cells arrested in tiers 1 and 2, and the nodules they observed only microscopically in postmortem examinations were neurons in the Arc tiers 3 and 4. Following this concept, the 


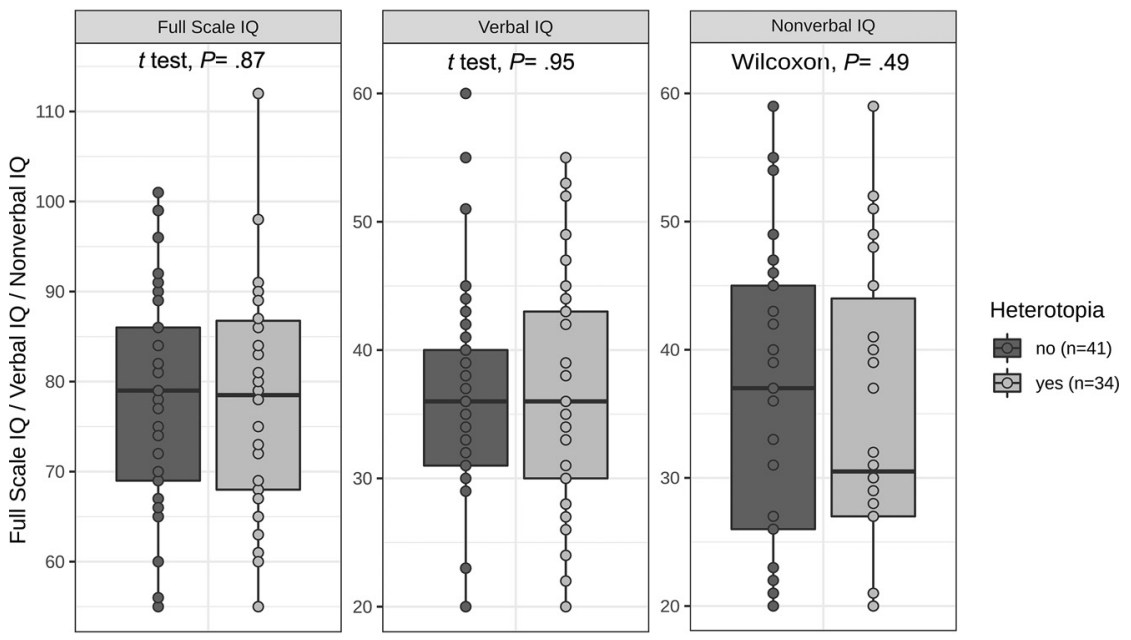

FIG 2. Association between the occurrence of heterotopia and IQ.

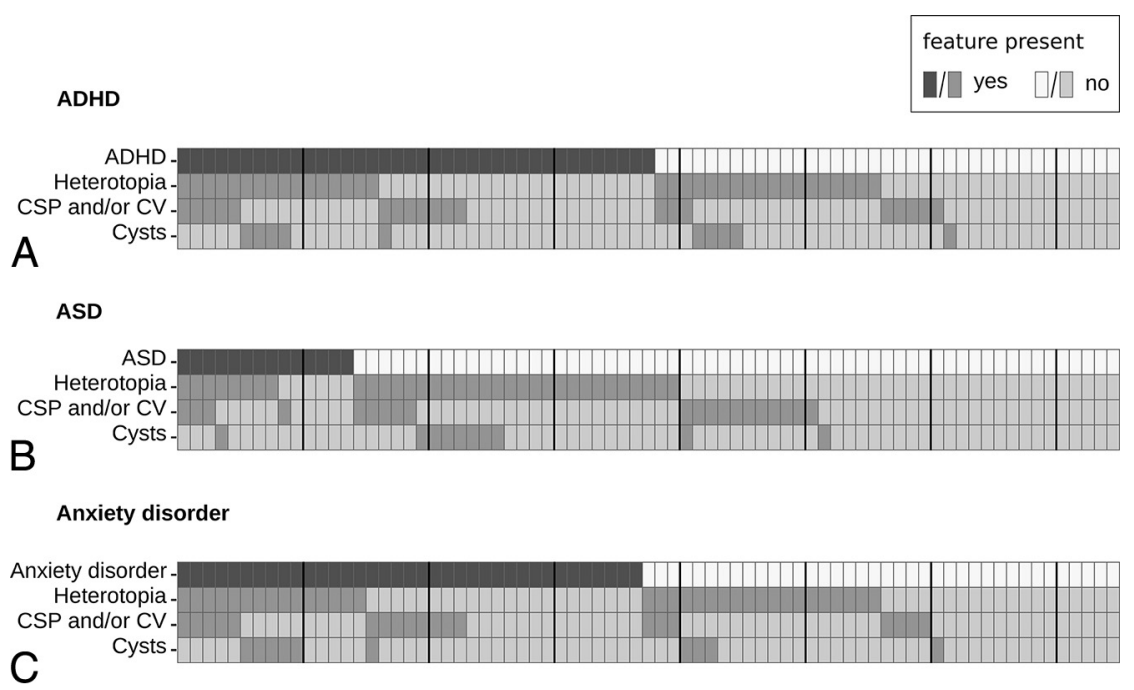

FIG 3. Pattern of distribution of the most frequent morphologic findings by psychiatric diagnosis. Every column represents 1 individual with 22q11.2DS. Ten individuals at a time are separated by a black line, and the shadings are coding the presence or absence of a finding, whereby the first row depicts the presence or absence of $\operatorname{ADHD}(A), \operatorname{ASD}(B)$, or anxiety disorder (C). Diagnosis of psychosis is not depicted due to the small number of subjects $(n=4)$.

heterotopia in the white matter that we found on MR imaging might represent larger chains of arrested Arc cells in tiers 3 and 4 . In addition to the spatial overlap of Arc cells and heterotopia in individuals with 22q11.2DS, there are also immunohistochemical features supporting the hypothesis of Rezazadeh et al.

Our observation of heterotopia in the white matter is limited because we had only 3D T1WI and low-resolution T2*WI at our disposal. Notably, in addition to the signal (cortex-isointense in both T1WI and T2* WI), we also considered the shape (round or ovoid). Furthermore, despite blinded observation, we found no heterotopia in the white matter in controls. The distribution pattern of the heterotopia-like lesions in controls was less concentrated in the frontal lobe than in individuals with 22q11.2DS, and in all 4 of these individuals, the lesions were isointense to the CSF rather than to the cortex in $\mathrm{T} 2{ }^{\star} \mathrm{WI}$, suggesting that these are probably enlarged perivascular spaces. Nevertheless, we cannot exclude some heterotopia in the white matter being nonspecific white matter lesions or enlarged perivascular spaces.

$\mathrm{PNH}$ are frequently associated with drug-resistant epilepsy. ${ }^{13}$ They often have intrinsic epileptogenicity but may not always be primarily involved in the generation of seizures. Both the heterotopic lesion and particularly the normotopic cortex are involved in the epileptogenic network. $^{22}$ Seizures seem to result from complex interactions between $\mathrm{PNH}$ and the allo- or neocortex. ${ }^{23}$ Ten percent of individuals with 22q11.2DS have a diagnosis of epilepsy. ${ }^{3}$ In the study of Rezazadeh et al, ${ }^{10} 6$ of the 7 individuals with 22q11.2DS with $\mathrm{PNH}$ had a history of seizures, which suggests that seizures could be the result of PNH. Information concerning epilepsy diagnosis of the subjects in our cohort was unsuitable for use in further analyses.

In $13 \%$ of the individuals with 22q11.2DS, we found periventricular cysts, especially adjacent to the anterior horns. Such cysts have been described only in single subjects with 22q11.2DS so far. ${ }^{19,24}$ The localization of the cysts is remarkably similar to the localization of PNH, and both seem to occur more often in combination. A reason for the occurrence of cysts could be a vulnerability of the microstructure due to the disturbed Arc cells migration.

We observed another migration disorder, polymicrogyria, in $4 \%$ of individuals with 22q11.2DS $(n=3)$ and none of the controls. This corresponds to the reported prevalences between $1 \%{ }^{25}$ and $7 \% .{ }^{4}$ Polymicrogyria in all our subjects occurred unilaterally right in the peri-Sylvian region, which is in line with observations by Robin et al. ${ }^{11}$

Another morphologic finding in individuals with 22q11.2DS was persistent CSP and/or CV (27\%). In only 1 subject the CSP appeared isolated without $\mathrm{CV}$. CV is normally present in up to $30 \%$ of neonates but persists into adulthood in $<1 \%$ of individuals, while CSP can persist in up to $20 \%{ }^{26}$ The increased rates of $\mathrm{CSP} / \mathrm{CV}$ in individuals with 22q11.2DS are known from prior studies, which reported a prevalence from $19 \%{ }^{19}$ to $84 \% .{ }^{27}$ Often no precise distinction is made between CSP and CSP/CV. The mechanisms through which a CSP/CV is maintained are unclear. Consistent with studies that noted an increased 
incidence of CSP or CV in patients with schizophrenia compared with healthy subjects, ${ }^{28,29}$ there is evidence that CSP is associated with psychosis in individuals with 22q11.2DS. ${ }^{19,20}$ Schmitt et $\mathrm{al}^{19}$ also found a significant association between incidental white matter abnormalities and psychosis. These relationships to psychosis remain unclear in our study due to the young age of the individuals with 22q11.2DS (mean age, 15.5 years). However, the mean age of onset for schizophrenia in individuals with 22q11.2DS is around 20 years. ${ }^{30}$

We did not find any significant relationships between morphologic findings and psychiatric diagnoses or general cognitive performance, consistent with prior studies. ${ }^{19,20,27}$ While there was a significant IQ difference between individuals with 22q11.2DS and controls, as expected, no differences were observed between individuals with 22q11.2DS with different morphologic abnormalities.

The functions of the frontal lobe, where most of the morphologic findings were found, are not specifically assessed via IQ tests.

Overall, despite the above-mentioned limitations and the limited sample size for these subgroup analyses, these findings suggest that morphologic findings do not necessarily reflect poorer brain function.

\section{CONCLUSIONS}

Taken together, our findings, $\mathrm{PNH}$ and heterotopia in the white matter (possibly reflecting interrupted Arc cells migration), persistent CSP/CV, and periventricular cysts, give clues to the brain development disorder induced by 22q11.2DS.

Disclosures: Felix Rosenow-UNRELATED: Consultancy: UCB, Eisai GmbH, Arvelle Therapeutics, GW Pharmaceuticals, Comments: scientific advice; Grants/Grants Pending: Deutsche Forschungsgemeinschaft, Hessisches Ministerium für Soziales und Integration (Hessian Ministry for Social Affairs and Integration), Hessisches Ministerium für Wissenschaft und Kunst (Hessian Ministry of Higher Education, Research and the Arts), European Union, Bundesministerium für Bildung und Forschung (Federal Ministry of Education and Research), State of Hessen LandesOffensive zur Entwicklung Wissenschaftlich-ökonomischer Exzellenz (state offensive for the development of scientific and economic excellence) Program, Comments: research grants*; Payment for Lectures Including Service on Speakers Bureaus: UCB, GlaxoSmithKline, Arvelle Therapeutics, Eisai GmbH, Medilearn India, Comments: for scientific and Continuing Medical Education presentations; Payment for Development of Educational Presentations: Eisai GmbH, Comments: patient education material. Christine Ecker-RELATED: Grant: Deutsche Forschungsgemeinschaft, Comments: Deutsche Forschungsgemeinschaft research grant.* Carrie E. BeardenRELATED: Grant: National Institute of Mental Health, Comments: A National Institute of Mental Health grant supported the recruitment and scanning costs of study participants*; UNRELATED: Board Membership: Neuroscience Novartis, Comments: Scientific Advisory Board member. *Money paid to the institution.

\section{REFERENCES}

1. McDonald-McGinn DM, Sullivan KE, Marino B, et al. 22q11.2 deletion syndrome. Nat Rev Dis Primers 2015;1:15071 CrossRef Medline

2. Niklasson L, Rasmussen P, Óskarsdóttir S, et al. Autism, ADHD, mental retardation and behavior problems in $\mathbf{1 0 0}$ individuals with 22q11 deletion syndrome. Res Dev Disabil 2009;30:763-73 CrossRef Medline

3. Eaton CB, Thomas RH, Hamandi K, et al. Epilepsy and seizures in young people with 22q11.2 deletion syndrome: prevalence and links with other neurodevelopmental disorders. Epilepsia 2019;60:818-29 CrossRef Medline

4. Hopkins SE, Chadehumbe M, Crowley TB, et al. Neurologic challenges in 22q11.2 deletion syndrome. Am J Med Genet A 2018;176:2140-45 CrossRef Medline
5. Moss EM, Batshaw ML, Solot CB, et al. Psychoeducational profile of the 22q11.2 microdeletion: a complex pattern. J Pediatr 1999;134:19398 CrossRef Medline

6. Eliez S, Schmitt JE, White CD, et al. Children and adolescents with velocardiofacial syndrome: a volumetric MRI study. Am J Psychiatry 2000;157:409-15 CrossRef Medline

7. Bearden CE, van Erp TG, Dutton RA, et al. Mapping cortical thickness in children with 22q11.2 deletions. Cereb Cortex 2007;17:188998 CrossRef Medline

8. Villalón-Reina JE, Martínez K, Qu X, et al. Altered white matter microstructure in 22q11.2 deletion syndrome: a multisite diffusion tensor imaging study. Mol Psychiatry 2020;25:2818-31 CrossRef Medline

9. Kiehl TR, Chow EW, Mikulis DJ, et al. Neuropathologic features in adults with 22q11.2 deletion syndrome. Cereb Cortex 2009;19:15364 CrossRef Medline

10. Rezazadeh A, Bercovici E, Kiehl TR, et al. Periventricular nodular heterotopia in 22q11.2 deletion and frontal lobe migration. Ann Clin Transl Neurol 2018;5:1314-22 CrossRef Medline

11. Robin NH, Taylor CJ, McDonald-McGinn DM, et al. Polymicrogyria and deletion 22q11.2 syndrome: window to the etiology of a common cortical malformation. Am J Med Genet A 2006;140:A2416-25 CrossRef Medline

12. Bingham PM, Zimmerman RA, McDonald-McGinn D, et al. Enlarged Sylvian fissures in infants with interstitial deletion of chromosome 22q11. Am J Med Genet 1997;74:538-43 CrossRef Medline

13. Battaglia G, Chiapparini L, Franceschetti S, et al. Periventricular nodular heterotopia: classification, epileptic history, and genesis of epileptic discharges. Epilepsia 2006;47:86-97 CrossRef Medline

14. Lin A, Ching CR, Vajdi A, et al. Mapping 22q11.2 gene dosage effects on brain morphometry. J Neurosci 2017;37:6183-99 CrossRef Medline

15. Lord C, Risi S, Lambrecht L, et al. The autism diagnostic observation schedule-generic: a standard measure of social and communication deficits associated with the spectrum of autism. J Autism Dev Disord 2000;30:205-23 CrossRef Medline

16. Lord C, Rutter M, Le Couteur A. Autism Diagnostic InterviewRevised: a revised version of a diagnostic interview for caregivers of individuals with possible pervasive developmental disorders. $J$ Autism Dev Disord 1994;24:659-85 CrossRef Medline

17. Core Team R. R: A Language and Environment for Statistical Computing. R Foundation for Statistical Computing; 2019

18. Wither RG, Borlot F, MacDonald A, et al. 22q11.2 deletion syndrome lowers seizure threshold in adult patients without epilepsy. Epilepsia 2017;58:1095-101 CrossRef Medline

19. Schmitt JE, Yi JJ, Roalf DR, et al. Incidental radiologic findings in the 22q11.2 deletion syndrome. AJNR Am J Neuroradiol 2014;35:2186-91 CrossRef Medline

20. Chow EW, Mikulis DJ, Zipursky RB, et al. Qualitative MRI findings in adults with 22q11 deletion syndrome and schizophrenia. Biol Psychiatry 1999;46:1436-42 CrossRef Medline

21. Paredes MF, James D, Gil-Perotin S, et al. Extensive migration of young neurons into the infant human frontal lobe. Science 2016;354: aaf7073 CrossRef Medline

22. Pizzo F, Roehri N, Catenoix H, et al. Epileptogenic networks in nodular heterotopia: a stereoelectroencephalography study. Epilepsia 2017;58:2112-23 CrossRef Medline

23. Aghakhani Y, Kinay D, Gotman J, et al. The role of periventricular nodular heterotopia in epileptogenesis. Brain 2005;128:641-51 CrossRef Medline

24. Mitnick RJ, Bello JA, Shprintzen RJ. Brain anomalies in velo-cardiofacial syndrome. Am J Med Genet 1994;54:100-06 CrossRef Medline

25. McDonald-McGinn DM, Sullivan KE. Chromosome 22q11.2 deletion syndrome (DiGeorge syndrome/velocardiofacial syndrome). Medicine (Baltimore) 2011;90:1-18 CrossRef Medline

26. Tubbs RS, Krishnamurthy S, Verma K, et al. Cavum velum interpositum, cavum septum pellucidum, and cavum vergae: a review. Childs Nerv Syst 2011;27:1927-30 CrossRef Medline 
27. Beaton EA, Qin Y, Nguyen V, et al. Increased incidence and size of cavum septum pellucidum in children with chromosome 22q11.2 deletion syndrome. Psychiatry Res 2010;181:108-13 CrossRef Medline

28. DeLisi LE, Hoff AL, Kushner M, et al. Increased prevalence of cavum septum pellucidum in schizophrenia. Psychiatry Res 1993;50:193-99 CrossRef Medline
29. Galarza $M$, Merlo $A B$, Ingratta $A$, et al. Cavum septum pellucidum and its increased prevalence in schizophrenia: a neuroembryological classification. J Neuropsychiatry Clin Neurosci. 2004;16:41-46 CrossRef Medline

30. Bassett AS, Chow EW, AbdelMalik P, et al. The schizophrenia phenotype in 22q11 deletion syndrome. Am J Psychiatry 2003;160:1580-86 CrossRef Medline 